\title{
乌ु \\ Answer first: Applying the heuristic-analytic theory of reasoning to examine student intuitive thinking in the context of physics
}

\author{
Mila Kryjevskaia* \\ Department of Physics, North Dakota State University, Fargo, North Dakota 58105, USA \\ MacKenzie R. Stetzer ${ }^{\dagger}$ \\ Department of Physics and Astronomy \& Maine Center for Research in STEM Education, \\ University of Maine, Orono, Maine 04469, USA \\ Nathaniel Grosz \\ Department of Physics, North Dakota State University, Fargo, North Dakota 58105, USA
}

(Received 22 November 2013; published 28 July 2014)

\begin{abstract}
We have applied the heuristic-analytic theory of reasoning to interpret inconsistencies in student reasoning approaches to physics problems. This study was motivated by an emerging body of evidence that suggests that student conceptual and reasoning competence demonstrated on one task often fails to be exhibited on another. Indeed, even after instruction specifically designed to address student conceptual and reasoning difficulties identified by rigorous research, many undergraduate physics students fail to build reasoning chains from fundamental principles even though they possess the required knowledge and skills to do so. Instead, they often rely on a variety of intuitive reasoning strategies. In this study, we developed and employed a methodology that allowed for the disentanglement of student conceptual understanding and reasoning approaches through the use of sequences of related questions. We have shown that the heuristic-analytic theory of reasoning can be used to account for, in a mechanistic fashion, the observed inconsistencies in student responses. In particular, we found that students tended to apply their correct ideas in a selective manner that supported a specific and likely anticipated conclusion while neglecting to employ the same ideas to refute an erroneous intuitive conclusion. The observed reasoning patterns were consistent with the heuristic-analytic theory, according to which reasoners develop a "first-impression" mental model and then construct an argument in support of the answer suggested by this model. We discuss implications for instruction and argue that efforts to improve student metacognition, which serves to regulate the interaction between intuitive and analytical reasoning, is likely to lead to improved student reasoning.
\end{abstract}

DOI: 10.1103/PhysRevSTPER.10.020109

PACS numbers: 01.55.+b, 01.40.G-

\section{INTRODUCTION}

Conceptual difficulties have been the subject of intense examination by the physics education research (PER) community for well over 30 years [1,2]. A significant body of empirical and theoretical work has led to widespread acknowledgment that teaching by telling has profound limitations for developing conceptual understanding. The need to focus on students' initial ideas is now widely accepted. In addition, the findings from PER have been highly successful in guiding the development of effective instructional strategies. An emerging body of evidence, however, suggests that even after targeted instruction, many

\footnotetext{
*mila.kryjevskaia@ndsu.edu

†mackenzie.stetzer@maine.edu

Nathaniel.C.Grosz@my.ndsu.edu
}

Published by the American Physical Society under the terms of the Creative Commons Attribution 3.0 License. Further distribution of this work must maintain attribution to the author(s) and the published article's title, journal citation, and DOI. students still struggle to apply the type of analytical reasoning approaches necessary to systematically and productively analyze unfamiliar situations [3]. Indeed, it appears that many undergraduate physics students fail to build reasoning chains from fundamental principles even if they possess the required conceptual knowledge to do so. Instead, they often rely on a variety of intuitive reasoning strategies that have been documented in the literature [4-6]. If the development of reasoning skills is a critical outcome of physics instruction, then it is imperative to direct our efforts toward a careful examination of student reasoning approaches (both productive and unproductive) and the identification of factors that appear to suppress the application of correct reasoning approaches even when the requisite conceptual understanding is present. An important step in research on student reasoning is the development of methodologies that allow for the disentanglement of conceptual understanding and reasoning approaches.

As part of an ongoing research effort, we have been identifying and developing sequences of questions that allow us to do so effectively. In this article, we present 
results from three such sequences that reveal inconsistencies in student reasoning approaches. On these sequences, many students demonstrate that they possess both the requisite conceptual understanding and the accompanying skills needed to perform the required reasoning, yet they fail to construct this reasoning to arrive at a correct answer. In certain contexts, we find that students tend to "abandon" suitable formal reasoning in favor of reasoning that was (perhaps) more intuitively appealing at that moment. We interpret these experimental results using the heuristicanalytic theory of reasoning [7]. Last, we reflect on the instructional implications emerging from this study.

\section{THEORETICAL FRAMEWORK}

It is a common expectation that, after instruction, students will consciously and systematically construct chains of reasoning that start from established scientific principles and lead to well-justified predictions. When student performance on course exams does not reveal such patterns, it is often assumed that students either do not possess a suitable understanding of relevant physics or are unable to construct such inferential reasoning chains due to deficiencies in reasoning abilities [8]. Psychological research on thinking and reasoning, however, seems to suggest that in many cases thinking processes follow paths that are strikingly different from those outlined above. Dual-process theories suggest that there are two distinct processes involved in many cognitive tasks, largely known as process 1 and process 2 . The former supports reasoning that is quick, intuitive, and automatic, while the latter is slow, rule-based, analytical, and reflective [9]. In many cases, these two processes yield different responses. Below we describe the role of each process as well as the role of the interaction between these processes in reasoning and decision making. Although dual-process theories have been proposed simultaneously (and mostly independently) by researchers examining various aspects of mental duality in different areas of psychology, these theories mostly agree on the basic characteristics of the two processes. In the present study, we apply the extended heuristic-analytic theory of thinking and reasoning proposed by Evans [7]. This theory is most relevant to us because it was specifically designed to explain a particularly puzzling phenomenon related to reasoning: the logical competence demonstrated on one task is often not exhibited in the performance of another related task.

The extended heuristic-analytic theory of thinking and reasoning suggests that two types of cognitive processes are involved in building inferences: heuristic (process 1) and analytic (process 2); see Fig. 1.

The heuristic process is quick and subconscious. It is responsible for generating selective mental models [10] of a presented situation. According to Evans, people generate only one mental model at a time (singularity principle). The model arising from the heuristic process is based on an

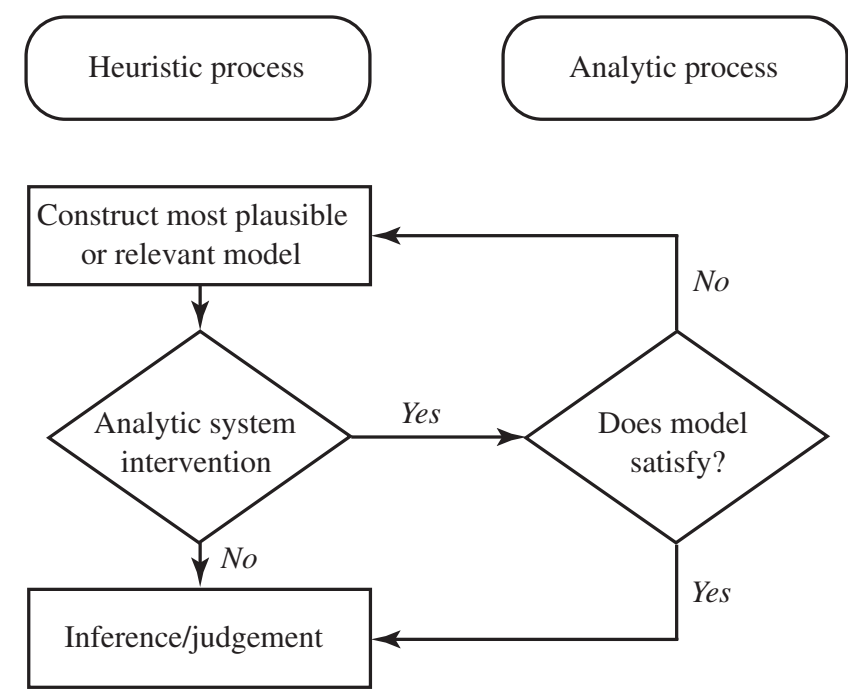

FIG. 1. Diagram illustrating reasoning process based on the heuristic-analytic model.

individual's most relevant prior knowledge and beliefs elicited by contextual cues and consistent with the individual's current goals (relevance principle); this model represents the most probable and believable description of the situation under consideration. A mental model arising from this heuristic process can be thought of as a person's "first impression." For example, when faced with the task of determining the probability of a specific outcome, people tend to use a mental shortcut called the availability heuristic, which is based on the notion that the easier it is to recall an event, the more frequently it must occur. For instance, people find it easier to recall words in the English language that start with a letter " $k$ " than those words that contain $\mathrm{k}$ in the third position. As a result, people tend to predict that there are fewer words in the latter category than there are in the former, while the opposite is actually true [11].

The content delivered by the heuristic process then becomes available for evaluation through a more explicit analytic process. This process operates based on the satisficing principle, according to which mental models "are evaluated with reference to the current goals and accepted if satisfactory" [7]. It is known, however, that in many cases heuristic processes can generate responses with little or no analytical intervention. If the analytical process is not engaged, the evaluation mechanism is circumvented and the first available, or default, model is used to generate a response. Factors that appear to increase the likelihood of analytical interventions include greater overall intelligence (cognitive abilities), the inclusion of implicit or explicit instructions for reasoners (e.g., there is an expectation of formal reasoning on an exam), and increased time on task. Motivation is also a relevant factor, since "people spend much more time and effort thinking about important decisions that carry strong implications for their personal 
goals" [7]. It is important to stress, however, that the engagement of the analytic process does not necessarily ensure that the desired chains of reasoning will be generated. While the heuristic process may yield biased responses based on first impressions, analytic processes may be impaired by somewhat different biases of their own. Specifically, the analytic process does not always employ a set of well-defined criteria for accepting or rejecting a default mental model. Indeed, people tend to believe that an idea is correct unless they find a good reason to think otherwise. They do not tend to spontaneously search for alternative mental models or counterarguments that could potentially falsify their original predictions. As such, even when the analytic process has been engaged, default first impression responses often still emerge.

In the present study, we use this heuristic-analytic theory of reasoning as a lens for investigating inconsistencies in student responses to physics problems. This work, therefore, contributes substantively to the research base on student reasoning in the context of authentic discipline-specific, contextually informed, learning-intensive scenarios. While we do not claim to be able to "observe" student reasoning paths directly, we draw inferences based on students' written responses and the degree to which their stated conclusions follow logically from the premises they express.

\section{RELEVANT PRIOR WORK}

Although dual-process theories are widely utilized in many areas of psychology, including those focusing on reasoning, decision making, and motivation, they have largely been ignored by physics education researchers focusing on related issues in the context of the learning and teaching of physics [12]. In a recent publication, however, Heckler drew attention to the applicability of these dual theories to the analysis of patterns of student reasoning in physics [8]. In addition to examining the influence of the automatic process (process 1) on student responses to science questions, he also described evidence from cognitive science for the existence of these two processes. Perhaps most importantly, Heckler provided a comprehensive overview of existing explanations for observed student reasoning patterns (including those focusing on misconceptions, knowledge in pieces or resources, and ontological categories), identified the limitations of each of these explanations, and constructed an argument for the applicability of dual-process theories of reasoning in providing a mechanism that both predicts and explains student preference for a specific line of reasoning.

Other studies relevant to our investigation probed student intuitive and formal lines of reasoning and examined student abilities to apply similar arguments under various conditions. Specifically, pilot studies by Sabella and collaborators suggested that poor student performance on assessment tasks may not simply stem from a lack of content knowledge $[13,14]$. Instead, the authors argued that many students possess the requisite formal knowledge and are able to activate it successfully in some situations, but they experience difficulties triggering that same knowledge in certain other contexts. Indeed, in such cases, students tend to activate intuitive rather than formal knowledge. For this reason, Sabella and colleagues argued that the interpretation of student performance on assessment tasks must extend beyond content understanding alone; many additional factors contribute to student performance on a given task. Lising and Elby argued that some student learning difficulties may stem, at least in part, from their epistemologies (i.e., ideas about knowledge and learning) [15]. Students may perceive a barrier between formal, technical reasoning and everyday, intuitive reasoning. This perception may prevent some students from searching for connections between these two seemingly disparate types of reasoning. Singh examined student performance on pairs (or sequences) of isomorphic problems (i.e., problems that require the same physics knowledge) [16]. Singh found that students were unlikely to recognize similarities between two isomorphic problems (e.g., one involving friction and one not involving friction) if the context of one of the situations triggered strong alternative ideas (e.g., the notion that static friction is always at its maximum value). Singh interpreted such findings from a knowledge in pieces perspective and argued that such strong alternative ideas may, in fact, leave students satisfied with their incorrect solution and prevent them from exploring productive analogies with other relevant situations.

\section{A. Comment on the definition of "intuitive"}

Physics instructors often stress that they want their students to develop physical intuition. It is usually assumed that the meaning of the phrase is immediately clear without an elaborate definition of the term "intuition." A closer analysis of the PER literature reveals that although various types of reasoning have been the focus of intense examination, to the best of our knowledge, no operational definitions of the terms intuition or "intuitive reasoning" exist. As Singh states, "Physical intuition is elusive-it is difficult to define, cherished by those who possess it, and difficult to convey to others. Physical intuition is at the same time an essential component of expertise in physics" [17]. The intuitive reasoning of experts, however, is distinctly different from that of novice learners; nevertheless, the same term is appropriate to characterize both.

When describing student reasoning, the term "intuitive knowledge" often represents knowledge that is not strictly based on content discussed in class; as such, intuitive can have a meaning opposite to that of "formal" knowledge (i.e., knowledge accepted by the physics community). For example, in order to articulate the difference between formal and intuitive reasoning, Sabella and Cochran note that poor student performance on a particular mechanics question may stem from student difficulties with the requisite formal reasoning (i.e., Newton's laws) or may 
be attributed to the use of an entirely different set of knowledge altogether, "possibly a more intuitive response, or a p-prim [...] - the knowledge associated with Newton's laws is not brought to the task" $[13,18]$. In the study by Lising and Elby [15], a schema for coding student interview data was designed in order to pinpoint when a student "used formal, classroom-taught reasoning versus 'everyday' and intuitive informal reasoning." The researchers tried to clarify these categories of reasoning by providing detailed discussions supplemented by multiple examples.

It is important to note that in the studies above, although formal and intuitive reasoning approaches were placed in two distinct categories, it was not suggested that a novice's intuitive or informal reasoning is undesirable or unhelpful (even in instances of erroneous student conclusions). We strongly agree with this view. As described earlier in this article, the heuristic system produces intuitive responses, for both experts and novices, that are quick and subconscious. Simon defines intuition as "nothing more and nothing less than the recognition" [19]. Kahneman builds on the work of Simon: "Valid intuitions develop when experts have learned to recognize familiar elements in a new situation and to act in a manner that is appropriate to it. [...] When confronted with a problem [...] the machinery of intuitive thought does the best it can. When an individual has relevant expertise, she will recognize the situation and the intuitive solution that comes into her mind is likely to be correct" [20]. For novices, the repertoire of relevant knowledge is much more limited; therefore, they are more likely to "recognize" a situation in an erroneous manner and the heuristic process may not draw upon the relevant knowledge, skills, or techniques. The goal of our study is thus to probe whether erroneous responses identified in inconsistent student reasoning patterns may be accounted for by the dual process theory of reasoning. In this article, we use the term intuitive reasoning to refer to student reasoning consistent with the first available response produced by the heuristic process. Such a response may not necessarily be limited to "everyday" knowledge; it may in fact utilize a variety of ideas that are ubiquitous in physics and physics instruction.

\section{OVERVIEW OF INVESTIGATION}

We feel that an important step in probing student reasoning in physics is the development of methodologies that allow for the disentanglement of conceptual understanding and reasoning approaches to the best of our abilities.

\section{A. Methodology}

For this study, we have drawn upon an emerging body of research in order to develop sequences of questions that allow us both (1) to separate those students who possess requisite knowledge and skills from those who do not and
(2) to examine, in detail, the reasoning approaches of those students who possess requisite knowledge and skills but fail to apply them in a coherent and consistent manner across all parts of the sequence. In order to determine whether a given student possessed the requisite knowledge and skills, we administered one or two "screening" questions that required the student to apply his or her understanding to arrive at a correct response. The following "target" question in each sequence featured an analogous situation that called for the application of the same knowledge and accompanying skills. We then examined the reasoning approaches employed on the target question by those students who answered the screening question correctly but failed to answer the target question correctly. Such question sequences were administered on two separate topics: parallel plate capacitors [21] and the transmission of waves at a boundary between two different media [22]. We identified patterns of student reasoning on these sequences and used the heuristic-analytic theory of reasoning to interpret inconsistencies in the reasoning approaches employed by students on the screening and target questions.

\section{B. Instructional context}

This study was conducted in two large enrollment introductory calculus-based physics courses for science and engineering majors at North Dakota State University (NDSU). The sequences of questions on which we will focus were included on course exams. Neither of the two courses included small-group discussion sessions. In one of the courses, however, the instructor implemented Tutorials in Introductory Physics, developed by the Physics Education Group at the University of Washington (UW) [23], in an interactive lecture format [24,25]. Instruction in the other course was fairly traditional and research-based materials were not used. In both courses, the majority of the students were also enrolled in a weekly two-hour laboratory.

\section{QUESTION SEQUENCES AND RESULTS}

Below, we discuss three different sequences of questions used in the present study: two focus on capacitors and one focuses on wave behavior at a boundary. For each sequence, we describe the questions along with the reasoning expected of students, present results of student performance, and interpret our findings in the context of heuristic-analytic theory. We also examine the performance of a single group of students across two different question sequences.

\section{A. Sequence of questions: Capacitors}

\section{Sequence overview}

In the capacitors sequence, students were shown two identically charged capacitors connected in series (see Fig. 2). Students were then told that the experimental setup 
A student charges identical parallel plate capacitors by connecting them one-by-one across a 9-volt battery. Each capacitor is carefully disconnected from the battery so that it is not discharged. The student then connects two of the identical capacitors in series, as shown at right. The capacitors are not connected to the battery.

Suppose that the left capacitor is modified: the distance between the plates is increased, as shown below right.

1. After the change, does the charge on the left capacitor increase, decrease or remain the same? Explain.

2. After the change, does the potential difference across the left capacitor increase, decrease or remain the same? Explain.

3. After the change, does the potential difference across the right capacitor increase, decrease or remain the same? Explain.

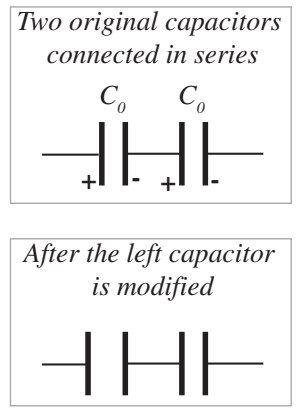

Two original capacitors

connected in series

left capacitor

FIG. 2. Sequence of questions: Capacitors. was modified by increasing the distance between the plates of the left capacitor. They were asked to determine how this modification affects (1) the charge $Q_{\text {left }}$ on the left capacitor, (2) the potential difference $\Delta V_{\text {left }}$ across the left capacitor, and (3) the potential difference $\Delta V_{\text {right }}$ across the right capacitor.

\section{Sequence results}

This sequence was included on a final exam administered to 174 students after tutorial-based interactive lecture instruction on parallel-plate capacitors. Approximately $50 \%$ of the students reasoned correctly on questions 1 and 2 that $Q_{\text {left }}$ remains the same, $C_{\text {left }}$ decreases, and therefore $\Delta V_{\text {left }}=Q_{\text {left }} / C_{\text {left }}$ must increase (see Table 1 ). However, only about half of these students were able to apply the same formal line of reasoning to the right capacitor in order to recognize that, since $Q_{\text {right }}$ and $C_{\text {right }}$ are unchanged, $\Delta V_{\text {right }}=Q_{\text {right }} / C_{\text {right }}$ must remain the same. Most students who analyzed the changes to the left and right capacitors inconsistently employed an argument commonly referred to as compensation reasoning, in which two physical quantities that change in opposite ways are assumed to cancel $[6,26,27]$. Many students articulated responses similar to the following:

"There is so much total potential across the two capacitors. Since the potential across the left capacitor increases, the potential across the right capacitor must decrease."

Others translated analogous arguments into a primarily symbolic form: $\uparrow \Delta V_{\text {left }}+\downarrow \Delta V_{\text {right }}=\Delta V_{\text {total }}$.

Many student responses in the compensation reasoning category contained elements of correct understanding. For example, one student wrote that $\Delta V_{\text {right }}$ "would increase, as the potential of the left capacitor drops, the right capacitor gains because it is not connected to the battery. If it was connected it would stay the same due to the constant potential being supplied by the battery." This student (and others) explicitly articulated the correct idea that the potential difference across a capacitor may change unless a battery is directly connected across the capacitor. When applied to the two-capacitor series network, this argument can be used to conclude that there is no reason why the potential difference across the network needs to be constant. However, the student failed to build on this correct idea in order to arrive at a correct answer. Instead, the correct idea was used to justify an erroneous conclusion.

\section{Discussion}

While half of the students responded to the first part correctly, many went on to "abandon" the appropriate formal reasoning in favor of reasoning that was (perhaps) more intuitively appealing at that moment. The student application of intuitive rather than formal reasoning is consistent with the first-impression reasoning paths suggested by heuristic-analytic theory. According to the theory, students first develop a mental model based on contextual cues, prior knowledge, and experiences. Such mental models may seem to be intuitively appealing to the students at the time. Indeed, the ideas of "equilibrium" or "conservation" are ubiquitous in introductory physics courses. Therefore, in accordance with the availability heuristic, those ideas are more likely to emerge before any others. For those students who did engage in analytical processes, these "first-impression" mental models may, in turn, have cued the use of equations embodying compensation 
TABLE I. Student performance on the capacitors sequence. (All reported percentages are percentages of total.)

\begin{tabular}{|c|c|c|c|c|c|c|c|c|c|}
\hline & \multicolumn{3}{|c|}{$\begin{array}{c}\text { Tutorial-based interactive lecture } \\
\text { instruction }\end{array}$} & \multicolumn{6}{|c|}{ Traditional lecture instruction } \\
\hline & \multicolumn{6}{|c|}{$\begin{array}{c}\text { Original sequence } \\
\text { (students arrive at an answer) }\end{array}$} & \multicolumn{3}{|c|}{$\begin{array}{l}\text { Modified sequence } \\
\text { (students justify a correct answer) }\end{array}$} \\
\hline \multirow{4}{*}{$\begin{array}{l}\text { Student performance } \\
\text { on screening questions } \\
\text { (percentage correct) }\end{array}$} & Q1 & \multicolumn{2}{|c|}{ Q2 } & Q1 & \multicolumn{2}{|c|}{ Q2 } & Q1 & \multicolumn{2}{|l|}{ Q2 } \\
\hline & $65 \%$ & \multicolumn{2}{|c|}{$55 \%$} & $43 \%$ & \multicolumn{2}{|c|}{$26 \%$} & \multicolumn{3}{|c|}{$41 \%$} \\
\hline & \multicolumn{3}{|c|}{ Both screening questions } & \multicolumn{3}{|c|}{ Both screening questions } & \multicolumn{3}{|c|}{ Both screening questions } \\
\hline & \multicolumn{3}{|c|}{$52 \%$} & \multicolumn{3}{|c|}{$24 \%$} & \multicolumn{3}{|c|}{$27 \%$} \\
\hline \multirow{2}{*}{$\begin{array}{l}\text { Performance on target } \\
\text { question of those } \\
\text { students who answered } \\
\text { both screening } \\
\text { questions correctly }\end{array}$} & $\begin{array}{l}\text { Correct } \\
\text { formal } \\
\text { reasoning }\end{array}$ & $\begin{array}{c}\text { Compensation } \\
\text { reasoning }\end{array}$ & Other & $\begin{array}{l}\text { Correct } \\
\text { formal } \\
\text { reasoning }\end{array}$ & $\begin{array}{c}\text { Compensation } \\
\text { reasoning }\end{array}$ & Other & $\begin{array}{l}\text { Correct } \\
\text { formal } \\
\text { reasoning }\end{array}$ & $\begin{array}{c}\text { Compensation } \\
\text { reasoning }\end{array}$ & Other \\
\hline & $25 \%$ & $24 \%$ & $3 \%$ & $12 \%$ & $12 \%$ & $0 \%$ & $23 \%$ & $1 \%$ & $3 \%$ \\
\hline
\end{tabular}

reasoning and prevented the students from applying their correct ideas about potential difference appropriately. In student responses to the capacitors sequence, correct ideas were often used to justify an erroneous conclusion. It appears that students applied their correct ideas in a selective manner (i.e., to the single capacitor) that supported a specific and likely anticipated conclusion while neglecting to employ the same ideas in a slightly different manner (i.e., to the two-capacitor network) that would refute an erroneous intuitive conclusion. Therefore, this pattern is consistent with heuristic-analytic theory in that many students develop a first-impression mental model (perhaps cued by the intuitive notion of equilibrium) and then construct an argument in support of the answer suggested by this model.

\section{B. Modified sequence of questions: Capacitors}

\section{Sequence overview}

In a modified version of the capacitors sequence, the correct answer to the third question (concerning a possible change in $\Delta V_{\text {right }}$ ) was provided and students were asked to explain why this answer is correct.

\section{Sequence results}

Both the modified and the original versions were administered in a single introductory calculus-based physics course that employed fairly traditional instruction. The versions were included on the same course exam, and roughly half of the students received the original version $(N=68)$ while the other half received the modified version $(N=78)$ [28].

After traditional instruction, the fraction of the students who demonstrated their conceptual understanding of capacitors in series by giving correct responses to both screening questions was lower than that after tutorial-based lecture instruction. Indeed, on the original version of the sequence, about a quarter of the students who received this version answered both screening questions correctly with correct reasoning, as indicated in Table I. However, only half of these students were able to provide analogous formal reasoning on the target question, while the other half used compensation reasoning. It is important to note that this distribution of formal and intuitive student responses on the target question is consistent with that obtained from students after tutorial-based lecture instruction. This result seems to suggest that such a distribution of student responses is unlikely to be the result of a specific instructional approach, but rather appears to be related to more general reasoning tendencies.

On the modified version of the sequence, student performance on the screening questions was similar to that on the original version: about a quarter of the students provided correct answers with correct reasoning to both screening questions. However, student performance on the target question was quite different. Almost all of the students who successfully answered the screening questions were also able to justify the answer provided for the target question correctly.

\section{Discussion}

A direct application of heuristic-analytic theory to student performance on the modified sequence suggests that a known answer to the target question cued more productive heuristic processes that ultimately yielded mental models consistent with correct formal reasoning. Thus, most students were able to supply the correct justification for the answer. This result is consistent with biases in reasoning described by the heuristic-analytic theory: when it is believed that an answer is true, the reasoning constructed to support that answer is also believed to be true and remains unchallenged. Indeed, our findings suggest that, at least on certain types of questions, students may be more successful at justifying 
an answer than at building a coherent reasoning path that leads to a correct prediction.

We recognize that a key limitation of our study is that we are only able to analyze the "final products" of student thinking, namely, student written responses to the exam questions. As such, we have no means of judging whether or not a mental model that eventually yielded a correct justification was the first and immediate model cued by the (provided) correct answer. It is also possible that the analytic process was more efficient in identifying and reconciling inconsistencies between first-impression mental models (based on "equilibrium") and the known answer, thus facilitating iterations in students' thinking processes which eventually resulted in revisions of their mental models and lines of reasoning.

\section{Sequence of questions: Pulse at a junction between two different springs}

The following sequence of questions, which features the somewhat more concrete context of mechanical wave pulses on a spring, was administered to students in order to investigate two related issues. First, we wanted to examine the extent to which students employ intuitive reasoning that leads to inconsistent conclusions when answering questions on topics other than capacitance. Second, we wanted to see if the tendency to use a particular class of reasoning approaches (e.g., intuitive or formal) on the target question may be attributed to individual student thinking strategies in general; if so, one might expect that a student who uses an intuitive approach on the target question in the capacitors sequence will also use an intuitive approach on the target question in the pulse at a junction sequence described below.

\section{Sequence overview}

In a sequence of questions about a pulse at a boundary between two different springs, students were asked to consider an experiment in which two different springs are connected at junction $J$ as shown in Fig. 3. In experiment 1 , a student generates a pulse on the left spring by moving his hand back and forth during a time $\Delta t_{0}$. In experiment 2 , a single change is made to experiment 1 such that the width of the generated pulse is doubled while the tension in the left spring and the time it takes to generate the pulse remain the same. In the screening question, students were asked to determine the change made in experiment 2; in the target question, students were asked to predict how this change affected the width of the transmitted pulse in experiment 2 .

In order to answer the first question correctly, students must recognize that the width of a pulse is determined by the time it takes to generate the pulse and by the propagation speed of the pulse in a spring. According to the propagation model typically used in introductory courses (i.e., waves of small amplitude traveling through nondispersive media), the propagation speed in a spring depends on the tension $(T)$ and the linear mass density $(\mu)$ via the relationship $v=\sqrt{T / \mu}$. Since both $\Delta t_{0}$ and $T$ remain the same in experiment 2 , in order to generate a pulse of greater width, the left spring must be replaced with

In experiment 1 , two different springs are connected at a junction point $J$. A student generates a pulse on the left spring shown below. It takes student's hand a time $\Delta t_{0}$ to quickly move the end of the spring back and forth in order to generate the pulse. In experiment 1 , the propagation speed of a pulse on the left spring is 1.5 times that on the right spring $\left(v_{L}=1.5 v_{R}\right)$.

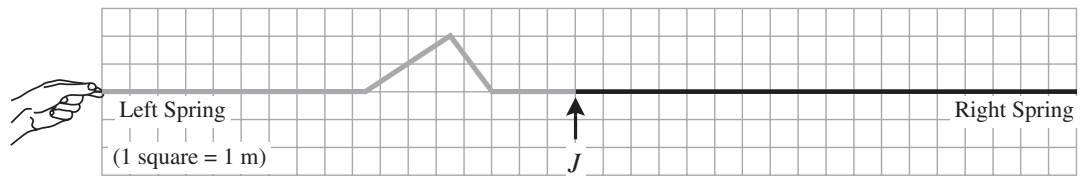

In experiment 2, a single change is made to experiment 1 . As a result of this change, the width of the generated pulse is doubled. The tension in the spring on the left and the time it takes for the student's hand to move to create the pulse is the same in both experiments. The spring on the right is unchanged.

1. Determine the change that has been made in experiment 2. Explain.

2. Compare the widths of the transmitted pulses on the right springs in experiment 1 and experiment 2. Is the width of the transmitted pulse on the right spring in experiment 2 greater than, less than, or equal to the width of the transmitted pulse on the right spring in experiment 1? If the width is not the same, determine the factor by which it is changed. Explain.

FIG. 3. Sequence of questions: Pulse at a junction between two different springs. 
a lighter spring $\left(\mu_{2}=\mu_{1} / 4\right)$, such that the propagation speed in the left medium is doubled.

In order to determine how the change in width of the incident pulse affects the shape of the transmitted pulse, students must apply an analogous argument: neither $\Delta t_{0}$ nor the right medium is changed, therefore the width of the transmitted pulse must remain the same.

\section{Sequence results}

This sequence was administered on a final exam after tutorial-based lecture instruction $(N=169)$. Approximately $75 \%$ of the students correctly recognized that a change in the left medium is responsible for the observed change in width of the incident pulse (see Table II). Only about $57 \%$ of all students were able to answer this target question correctly with correct reasoning. While almost half of the students answered both questions correctly (52\%), approximately a quarter of those students who answered the screening question correctly (thereby demonstrating that they possess the requisite formal knowledge) applied intuitive rather than formal arguments to the target question. In particular, the most prevalent incorrect student argument stemmed from the idea that the width of the transmitted pulse depends on the width of the incident pulse; since the width of the incident pulse is doubled, these students argued that the width of the transmitted pulse must double as well. For example, one student stated the following:

"Ex $2>\operatorname{Ex} 1$ by the factor of 2 since the width of the transmitted is dependent on the width of the incident and the incident is doubled, then the transmitted will be doubled as well."

This student, as well as others who provided similar arguments, did not justify his intuitive notion of the dependence of the width of the transmitted pulse on the width of the incident pulse. However, those students who did attempt to justify this notion applied other types of intuitive arguments. Some students stated that the propagation speed of the transmitted pulse depends on that of the incident pulse: since the incident pulse travels faster in

TABLE II. Student performance on the pulse at a junction between two different springs sequence. (All reported percentages are percentages of total.)

\begin{tabular}{l|c|c|c}
\hline \hline & \multicolumn{2}{|c}{$\begin{array}{c}\text { Tutorial-based interactive lecture } \\
\text { instruction }\end{array}$} \\
\hline $\begin{array}{c}\text { Student performance on } \\
\text { screening question } \\
\text { (percentage correct) }\end{array}$ & \multicolumn{3}{|c}{$75 \%$} \\
\hline $\begin{array}{c}\text { Performance on target } \\
\text { question of those students } \\
\text { who answered screening } \\
\text { question correctly }\end{array}$ & $\begin{array}{c}\text { Correct with } \\
\text { correct } \\
\text { reasoning }\end{array}$ & $\begin{array}{c}\text { Intuitive } \\
\text { reasoning }\end{array}$ & Other \\
\cline { 2 - 4 } & $52 \%$ & $18 \%$ & $5 \%$ \\
\hline \hline
\end{tabular}

experiment 2, the transmitted pulse must be traveling faster as well; as such, the width of the transmitted pulse must increase. A small fraction of students attempted to construct a line of reasoning based on the pulse behavior at the junction and provided an intuitive argument similar to the following:

"The wavelength was changed on the generated pulse so that when the longer pulse gets to the junction it will have more time to stretch out."

This argument, which may originate from a primitive notion that "more means more," [18] is inconsistent with a formal understanding of pulse behavior in two different media. Indeed, the time it takes to generate the pulse is unchanged in experiment 2; therefore, the time it takes for the pulse to pass by a point in either medium (including the junction) also must remain unchanged. Since neither the generation time nor the speed of the transmitted pulse has changed, its width must also remain unchanged.

\section{Discussion}

Results from this question sequence also appear to be consistent with the heuristic-analytic theory of reasoning. A fraction of those students who correctly recognized that the increased width of the incident pulse must be attributed to a change in the left medium did not apply the same knowledge to the analysis of the width of the transmitted pulse. These students failed to reason that since neither the right medium nor the source is changed, the shape of the transmitted pulse must remain the same. Instead, it appears that the heuristic process suggested a default model consistent with the documented student tendency to think of waves as objects [5]. Observations that the initial state of an object determines its final state are abundant in everyday life. Indeed, a ball that moves faster on a sidewalk will result in a ball that moves faster once it reaches the lawn. This notion, when extended to the context of waves at a boundary, leads to a similar model: the propagation speed of a transmitted wave depends on that of the incident wave. Other properties of pulses (or waves), such as width (or wavelength), may also be perceived to obey an analogous intuitive rule.

Most students who provided responses consistent with this objectlike wave model did not provide formal justifications for statements such as " $v_{f}$ (or $\lambda_{f}$ ) depends on $v_{i}$ (or $\left.\lambda_{i}\right)$." It appears that, as in the case of the capacitors sequence, students did not search for alternative models, perhaps due to the strongly perceived credibility of the firstimpression models.

\section{Examination of impact of individual student thinking styles on reasoning}

In addition to exploring the utility of the heuristicanalytic theory as a framework for the examination of 
inconsistencies in student reasoning approaches in physics, we were interested in probing whether or not students' tendency to apply intuitive rather than formal reasoning can be attributed to individual differences in thinking styles. Evans suggests that there is ample evidence for two types of thinkers: intuitive and analytical [7]. Intuitive thinkers, when presented with an unfamiliar situation, are largely engaged in heuristic processing of information, which is automatic, mostly subconscious (or implicit), relatively fast, and based on experience and association. Analytical thinkers, on the other hand, are characterized by information processing that is controlled, explicit, relatively slow, rational, and based on rules and underlying principles [29].

In this study, both the original capacitors sequence and the pulse at a junction sequence discussed above were administered to the same students in a tutorial-based lecture course. Of those students who answered the screening questions associated with both sequences correctly $(N=63)$, their responses to the target questions in both sequences were categorized and examined for consistency in terms of the reasoning approaches employed. About $44 \%$ of these students consistently provided (correct) formal reasoning on the target questions in both sequences. Another $13 \%$ of the students consistently provided intuitive reasoning when responding to the target questions in both sequences. Perhaps most importantly, approximately $43 \%$ of students who answered the target question on one sequence correctly applied intuitive reasoning on the other target question. (See Table III.)

Based on these data alone, we are not able to attribute formal and intuitive student response patterns to general differences in student thinking styles. Indeed, some "intuitive thinkers" on the capacitors task may be classified as "analytical thinkers" on the pulse at a junction task and vice versa. We recognize, however, that an analysis of individual student reasoning patterns on these two tasks alone does not provide adequate information for building a "thinking profile" for each student. Nevertheless, we are inclined to think that the boundaries between the two categories of thinkers emerging from previous clinical studies may be less defined in the context of authentic,

TABLE III. Patterns of reasoning on two question sequences: capacitors and pulse at a junction between two different springs. Only those students who demonstrated (on the screening questions) that they possessed the formal knowledge necessary to answer these sequences correctly are considered $(N=63)$.

\begin{tabular}{lccc}
\hline \hline & \multicolumn{3}{c}{$\begin{array}{c}\text { Pulse at } \\
\end{array}$} \\
& Capacitors & a junction & Both \\
\hline $\begin{array}{l}\text { Correct with correct reasoning } \\
\text { on all parts }\end{array}$ & $56 \%$ & $76 \%$ & $44 \%$ \\
$\begin{array}{l}\text { Intuitive reasoning on } \\
\text { target question }\end{array}$ & $44 \%$ & $24 \%$ & $13 \%$ \\
\begin{tabular}{l} 
Correct on one task, intuitive on the other \\
\hline \hline
\end{tabular} & & $43 \%$ \\
\hline \hline
\end{tabular}

discipline-specific learning environments. It could be argued that the majority of students whose responses were examined in this study are in a state of transition, as they gradually develop from novices into experts. As such, the observed pattern of "mixed" reasoning approaches applied by a large fraction of these students is rather consistent with the probabilistic nature of student responses outlined by Bao and Redish [30]. We therefore suspect that, in the context of an authentic learning environment, it is more productive to analyze student reasoning in terms of noviceand expertlike characteristics, an approach that is more closely aligned with the transitional nature of learning.

\section{ADDRESSING THE LIMITATIONS OF HEURISTIC-ANALYTIC THEORY IN EFFORTS TO IMPROVE INSTRUCTION}

The heuristic-analytic theory of reasoning suggests that initial mental models of both novice learners and experts are intrinsically implicit and experience driven. Experts, however, draw on a more sophisticated body of formal knowledge and on a wealth of experience with the relevant content. Despite these differences, in both cases, it is the function of the analytic process to evaluate the firstimpression model. The engagement of the analytic process is therefore critical for building productive reasoning chains based on formal knowledge and fundamental principles. However, as stated earlier, the analytic process continues to be influenced by reasoning biases consistent with the satisficing principle. As such, novice learners typically only consider ideas that are supportive of their default mental model. They often do not consider alternative scenarios and are easily satisfied by a default model provided it appears to "be good enough" and/or "makes sense." In such cases, the role of the analytic process is then to rationalize the conclusions that follow. The analytic process of experts, on the other hand, functions in a different, more productive manner. Experts are well aware of their reasoning approaches, both productive and unproductive. For them, thinking is explicit. Indeed, experts tend to (1) scrutinize reasoning paths (thus becoming aware of biases and shortcuts in their reasoning), (2) identify strengths and limitations in their understanding, (3) continuously reflect upon their own thinking, and (4) actively seek and identify ways to improve their understanding in order to successfully complete tasks [31].

\section{A. Limitations of heuristic-analytic theory}

For both researchers and instructors, it is vital to understand the mechanisms responsible for novices' inconsistent and often erroneous reasoning approaches. In this paper, we have made a case for the utility of heuristic-analytic theory in providing a robust framework for understanding these mechanisms. At the same time, however, we recognize the significant limitations of the theory in providing a 
framework for the development of instructional strategies intended to facilitate the gradual transition from novicelike to expertlike reasoning habits. Indeed, although the concept of metacognition plays a critical role in the enhancement of student reasoning, it is not explicitly included in the heuristic-analytic theory of reasoning. Below we present a brief overview of metacognition and stress the importance of the concept to any efforts aimed at improving student reasoning.

\section{B. Overview of metacognition}

This "hidden level" of behavior characteristic of expert learners is often described as "thinking about thinking," or metacognition. We argue that individuals' metacognitive abilities are responsible for the regulation of the thinking process through productive engagement of the analytic process. (Support for this argument is also found in the psychology literature [32].) Indeed, research suggests that effective thinkers possess a diverse repertoire of metacognitive abilities that they consciously deploy to support and guide their reasoning.

Researchers have identified two broad aspects of metacognition: (1) knowledge about productive thinking strategies (e.g., knowing of and about specific practices that are likely to facilitate progress on challenging tasks) and (2) implementation of productive thinking strategies to regulate effort (e.g., what one actually does while working on a challenging task) [33]. The distinction between these two aspects makes clear that knowledge alone does not ensure that metacognition will be actively used to support intellectual progress. A particularly illustrative analogy involves lifestyle practices that support physical health: knowing that exercise and a well-balanced diet contribute to health does not ensure that an individual will exercise or eat right. Therefore, simply lecturing on the importance of carefully examining reasoning and problem-solving practices is not enough. Rather, the implementation of instruction that explicitly targets students' monitoring and self-evaluation skills is more likely to promote the development of metacognitive skills.

There are several specific functions of metacognition that we feel are critical to the improvement of reasoning in physics. Awareness of one's own thinking involves the ability of learners to tell an explicit "story" of how they came to understand what they currently understand (e.g., what steps in the development of ideas allowed them to arrive at a specific conclusion). Reflection on one's own learning may also promote recognition of the applicability of reasoning strategies to specific situations. This skill is critical for matching reasoning approaches to the tasks for which they are best suited. These two skills highlighted thus far are of key importance because they motivate students to change their thinking habits and learning routines and serve as the foundation upon which the development of more sophisticated reasoning skills may occur. Evaluating, monitoring, and planning skills overlap in many ways and enable effective learners to recognize when they do or do not understand something and what learning steps are needed to improve their understanding. The primary functions of this set of skills are to analyze current thinking, assess the robustness of mental models, and suggest further actions.

\section{Role of metacognition in instruction that targets student reasoning}

Adopting new thinking strategies is complex and demanding for novice learners, but the process can be facilitated by instructors who actively support the development of students' metacognitive skills. As Herscovitz et al. note, "It requires the development of new teaching strategies and, in most cases, changing instructors' and students' roles in the learning process" [33]. We share the view of many researchers who think that the development of metacognitive skills and thinking habits should be integrated into physics-specific instruction. Indeed, some instructional materials and/or approaches that target metacognition have already been developed by members of the PER community, including Open Source Tutorials [34,35], Investigative Science Learning Environment (ISLE) laboratories [36], and reflection activities implemented in the context of problem solving in algebra-based and calculusbased courses [37].

We speculate that sequences of questions similar to those highlighted in this study may be useful for enhancing instruction by targeting student reasoning [38]. Indeed, as part of new project to promote the development of student metacognitive abilities, we have been identifying (1) sets of questions that appear to be different but require similar reasoning, and (2) sets of questions that appear to be similar but require the application of different lines of reasoning. The original capacitors sequence is a particularly illustrative example of the former category. In this sequence, the screening questions and the target question require identical analyses of the relationship among $\Delta V$, $Q$, and $C$; for each capacitor, $Q$ remains the same, so $\Delta V$ only depends on the capacitance, and thus the state of the left capacitor is independent of that of the right capacitor. As an example of the latter category, one can imagine pairing the original capacitors task with a variant in which a battery is now connected across the series network of capacitors. (Indeed, we have observed that many students apply the same reasoning to both scenarios.) The role of the instructor is then to use these question sets to foreground student reasoning approaches and to help students "visualize their reasoning paths" by (1) explicitly articulating how, if at all, their first-impression models evolved and (2) identifying those factors that led to a specific line of reasoning or motivated substantive changes in reasoning. 


\section{CONCLUSION}

The goal of this study was to examine inconsistencies in student reasoning approaches and to explore the mechanisms responsible for such inconsistencies. To accomplish this, we first developed a methodology that involved the analysis of question sequences containing both screening and target questions in order to facilitate the disentanglement of student conceptual difficulties from specific reasoning approaches. Through the use of these screening questions, we were able to ensure that student constructed reasoning paths on the target question reflected specific reasoning processes and not simply deficiencies in conceptual understanding. We then identified considerable inconsistencies in student reasoning approaches employed on the screening and target questions. Indeed, a significant fraction of students who applied correct and complete reasoning on the screening question(s) failed to do so on a target question that called for the same knowledge and skills. We have shown that the heuristic-analytic theory of reasoning can be used to account for, in a mechanistic fashion, the observed inconsistencies in student responses. Given that the theory suggests that such inconsistencies arise from a failure to adequately engage the analytic process, we argue that efforts to improve student metacognition, which serves to regulate the interaction between heuristic-based and analytical approaches, will lead to improved student reasoning. As such, we anticipate that an explicit focus on metacognition and the development of metacognitive abilities in instruction will help bridge the gap between novicelike and expertlike reasoning.

\section{ACKNOWLEDGMENTS}

The authors wish to acknowledge contributions from and valuable discussions with Beth A. Lindsey, Paula R. L. Heron, Andrew Boudreaux, and Andrew F. Heckler. Support from the National Science Foundation under Grants No. DUE-0962805, No. DUE-1245999, and No. DUE-1245313 is also gratefully acknowledged.
[1] National Research Council, Adapting to a Changing World-Challenges and Opportunities in Undergraduate Physics Education, Committee on Undergraduate Physics Education Research and Implementation, Board on Physics and Astronomy (BPA), Division on Engineering and Physical Sciences (DEPS) (National Academies Press, Washington, DC, 2013).

[2] D. E. Meltzer and R. K. Thornton, Resource letter ALIP-1: Active-learning instruction in physics, Am. J. Phys. 80, 478 (2012).

[3] See, for example, M. E. Loverude, C. H. Kautz, and P. R. L. Heron, Student understanding of the first law of thermodynamics: Relating work to the adiabatic compression of an ideal gas, Am. J. Phys. 70, 137 (2002); H. G. Close and P. R. L. Heron, Research as a guide for improving student learning: An example from momentum conservation, ibid. 78, 961 (2010); M. Kryjevskaia, M. R. Stetzer, and P. R. L. Heron, Student understanding of wave behavior at a boundary: The limiting case of reflection from fixed and free ends, ibid. 79, 508 (2011).

[4] E. F. Redish, Oersted lecture 2013: How should we think about how our students think?, Am. J. Phys. 82, 537 (2014).

[5] M. Wittmann, R. N. Steinberg, and E. F. Redish, Making sense of how students make sense of mechanical waves, Phys. Teach. 37, 15 (1999).

[6] C. H. Kautz, P. R. L. Heron, P. S. Shaffer, and L. C. McDermott, Student understanding of the ideal gas law, Part II: A microscopic perspective, Am. J. Phys. 73, 1064 (2005).

[7] J. St. B. T. Evans, The heuristic-analytic theory of reasoning: extension and evaluation, Psychon. Bull. Rev. 13, 378 (2006).
[8] A. F. Heckler, The ubiquitous patterns of incorrect answers to science questions: The role of automatic, bottom-up processes, Psych. Learn. Motiv. 55, 227 (2011).

[9] K. Frankish, Dual-process and dual-system theories of reasoning, Philos. Compass 5/10, 914 (2010).

[10] P. N. Johnson-Laird, How We Reason (Oxford University Press Inc., New York, 2006).

[11] A. Tversky and D. Kahneman, Availability: A heuristic for judging frequency and probability, Cogn. Psychol. 5, 207 (1973).

[12] B. Geller, B. Dreyfus, J. Gouvea, V. Sawtelle, C. Turpen, and E. Redish, Like Dissolves Like: Unpacking Student Reasoning About Thermodynamic Heuristics, 2013 PERC Proceedings edited by P. V. Engelhardt, A. D. Churukian, and D. L. Jones (Portland, OR, 2013).

[13] M. Sabella and G. L. Cochran, Evidence of intuitive and formal knowledge in student responses: Examples from the context of dynamics, AIP Conf. Proc. 720, 89 (2004).

[14] E. Watkins and M. Sabella, Examining the effectiveness of clickers on promoting learning by tracking the evolution of student responses, AIP Conf. Proc. 1064, 223 (2008).

[15] L. Lising and A. Elby, The impact of epistemology on learning: A case study from introductory physics, Am. J. Phys. 73, 372 (2005).

[16] C. Singh, Assessing student expertise in introductory physics with isomorphic problems. II. Effect of some potential factors on problem solving and transfer, Phys. Rev. ST Phys. Educ. Res. 4, 010105 (2008).

[17] C. Singh, When physical intuition fails, Am. J. Phys. 70, 1103 (2002). 
[18] A. diSessa, Knowledge in pieces, in Constructivism in the Computer Age, edited by G. Forman and P. Pufall (Lawrence Erlbaum, Hillsdale, NJ, 1988).

[19] H. A. Simon, What is an "Explanation" of behavior?, Psychol. Sci. 3, 150 (1992).

[20] D. Kahneman, Thinking, Fast and Slow (Farrar, Straus and Giroux, New York, 2011).

[21] M. Kryjevskaia and M. R. Stetzer, Examining inconsistencies in student reasoning approaches, AIP Conf. Proc. 1513, 226 (2013).

[22] M. Kryjevskaia, M. R. Stetzer, and P. R. L. Heron, Student understanding of wave behavior at a boundary: The relationships among wavelength, propagation speed, and frequency, Am. J. Phys. 80, 339 (2012).

[23] L. C. McDermott and P. S. Shaffer, the Physics Education Group at the University of Washington, Tutorials in Introductory Physics, Preliminary Second Edition (Prentice Hall, Upper Saddle River, NJ, 2011).

[24] In the course with Tutorials implemented in a lecture-based format, modified tutorial worksheet questions were presented one by one on a screen and all students were asked to consider a given question at the same time. Those questions deemed critical for the development of ideas were converted to clicker questions, and students were asked to discuss their reasoning with one another prior to voting. Results of the class vote were then immediately revealed, and students were encouraged to justify their answers in a whole-class discussion. Key features of the canonical implementation of the UW tutorials such as a low student-to-instructor ratios, self-paced work in small groups, instructor feedback tailored to individual students, and opportunities for metacognition could not be implemented in the instructional environment at NDSU. For a complete discussion of tutorial implementation in the lecture-based format, see Ref. [25].

[25] M. Kryjevskaia, A. Boudreaux, and D. Heins, Assessing the flexibility of research-based instructional strategies: Implementing, Tutorials in Introductory Physics in the lecture environment, Am. J. Phys. 82, 238 (2014).

[26] R. Lawson and L. C. McDermott, Student understanding of the work-energy and impulse-momentum theorems, Am. J. Phys. 55, 811 (1987).

[27] B. A. Lindsey, P. R. L. Heron, and P. S. Shaffer, Student ability to apply the concepts of work and energy to extended systems, Am. J. Phys. 77, 999 (2009).
[28] Analysis of student performance on all course exams as well as in the entire course suggest that the two groups of students are not significantly different.

[29] K. E. S. Stanovich and Richard F. West, Individual differences in reasoning: Implications for the rationality debate?, Behav. Brain Sci. 23, 645 (2000).

[30] L. Bao and E. F. Redish, Model analysis: Representing and assessing the dynamics of student learning, Phys. Rev. ST Phys. Educ. Res. 2, 010103 (2006).

[31] How People Learn: Brain, Mind, Experience, and School, edited by J. Bransford, A. Brown, and R. Cocking (National Academy Press, Washington, DC, 2000), expanded edition.

[32] E. Amsel, P. A. Klaczynski, A. Johnston, S. Bench, J. Close, E. Sadler, and R. Walker, A dual-process account of the development of scientific reasoning: The nature and development of metacognitive intercession skills, Cognit. Dev. 23, 452 (2008).

[33] O. Herscovitz, Z. Kaberman, L. Saar, and Y. J. Dori, The relationship between metacognition and the ability to pose questions in chemical education, in Metacognition in Science Education: Trends in Current Research, edited by A. Zohar and Y. J. Dori (Springer, New York, 2012).

[34] R. E. Scherr and A. Elby, Enabling informed adaptation: Open-source physics worksheets integrated with implementation resources, AIP Conf. Proc. 883, 46 (2007).

[35] T. I. Smith and M. C. Wittmann, Comparing three methods for teaching newton's third law, Phys. Rev. ST Phys. Educ. Res. 3, 020105 (2007).

[36] E. Etkina, S. Murthy, and X. Zou, Using introductory labs to engage students in experimental design, Am. J. Phys. 74, 979 (2006).

[37] See, for example, E. Yerushalmi, E. Cohen, A. Mason, and C. Singh, What do students do when asked to diagnose their mistakes? Does it help them? I. An atypical quiz context, Phys. Rev. ST Phys. Educ. Res. 8, 020109 (2012); What do students do when asked to diagnose their mistakes? Does it help them? II. A more typical quiz context, Phys. Rev. ST Phys. Educ. Res. 8, 020110 (2012); C. Henderson and K. A. Harper, Quiz corrections: Improving learning by encouraging students to reflect on their mistakes, Phys. Teach. 47, 581 (2009).

[38] A. Elby, Helping physics students learn how to learn, Am. J. Phys. 69, S54 (2001). 